\title{
ARTIGOS
}

\section{ESTUDO EPIDEMIOLÓGICO DE FONTES DE ALIMENTAÇÃO SANGÜÍNEA DOS TRIATOMÍNEOS DA FAZENDA AROEIRA (CATOLÉ DO ROCHA, PARAÍBA) E CIRCUNVIZINHANÇAS}

\author{
Carlos Brisola Marcondes*, João Carlos Pinto Dias, Leonardo A. Guedes, \\ Antenor N. Ferraz Filho, Vera L.C.C. Rodrigues e Delosmar D. Mendonça.
}

\begin{abstract}
Analisamos a fonte de alimentação sangüínea de 94 triatomíneos (51 Triatoma brasiliensis, 42 T. pseudomaculata e um Rhodnius neglectus) da Fazenda Aroeira, no Município de Catolé do Rocha, no Estado da Paraíba, e de quatro localidades próximas. Observamos positividade para marsupial, homem e ave, respectivamente, de 39,7, 6,2 e 23,0\%. Três insetos da Fazenda Aroeira, positivos para Trypanosoma cruzi, foram positivos para marsupial, só um deles também para outro mamífero. Os marsupiais são a mais importante fonte de T. cruzi para os insetos da área e estes têm pouco contato com o homem.

Palavras-chaves: Triatominae. Fonte de alimentação sangüínea. Semi-árido. Doença de Chagas. Nordeste do Brasil.
\end{abstract}

No final de 1986, ocorreu um surto de doença de Chagas, de provável contaminação oral, na Fazenda Aroeira, no Município de Catolé do Rocha, no noroeste da Paraíba ${ }^{78}$. Como parte dos estudos realizados na área, capturamos triatomíneos em vários biótopos da Fazenda e de quatro localidades vizinhas, duas das quais em outros municípios.

Os insetos foram examinados para infecção por T. cruzi e identificação de fonte de alimentação sangüínea, de modo a fornecer dados adicionais para o esclarecimento da epidemiologia do surto.

\section{MATERIAL E MÉTODOS}

O Município de Catolé do Rocha, com

\footnotetext{
Trabalho do Núcleo de Medicina Tropical da Universidade Federal da Paraỉa, João Pessoa, PB e da Superintendência de Controle de Endemias, Moji Guaçu, SP, apresentado na $17^{\text {a }}$ Reunião de Pesquisa Básica em Doença de Chagas, Caxambu, 1990.

* Bolsista do CNPq

Apoio financeiro da Finep (Proc. 43.86.0736.00).

Endereço para correspondência: Prof. Carlos Brisola Marcondes. Depto. de Microbiologia e Parasitologia/CCB/ UFSC. Campus Trindade, 8804 Florianópolis, SC.

Recebido para publicação em 28/08/89.
}

três localidades, e os de Jericó e Brejo dos Santos, com uma localidade cada, ficam na micro-região de Catolé do Rocha, com pluviosidade anual média de $800 \mathrm{~mm}$, concentrada na primeira metade do ano, e estão na categoria climática Awi de Koeppen ${ }^{10}$.

Os triatomíneos foram capturados em domicílios e anexos e em alguns biótopos afastados dos domicílios, como montes de pedras, usando pinças e, quando necessário, desalojante (Piriza CE a $2 \%$ ), por pessoal da Sucam, em novembro de 1986. A pesquisa foi exaustiva, incluindo insetos obtidos antes e após a aplicação de cipermetrina (Ripcord PM).

Os insetos foram examinados por compressão abdominal, para pesquisa de $T$. cruzi, e tiveram o restante do tubo digestivo espalhado em papel de filtro. Os blocos de papel de filtro foram enviados para a SUCEN, onde foram realizadas reações de preciptina pela técnica do tubo capilar, para oito hospedeiros (homem, marsupial, ave, roedor, cão, gato, cabra e lagarto).

As diferenças entre as positividades para as fontes e espécies foram avaliadas por teste de $\mathrm{X}^{2}$, considerando o nível de significância de $5 \%$.

\section{RESULTADOS}

Os resultados globais da área são 
Marcondes CB, Dias JCP, Guedes LA, Ferraz Filho AN, Rodrigues VLCC, Mendonça DD. Estudo epidemiológico de fontes de alimentação sangüinea dos triatomineos da Fazenda Aroeira (Catolé do Rocha, Paraíba) $e$ Circunvizinhanças. Revista da Sociedade Brasileira de Medicina Tropical 24:137-140, jul-set, 1991

apresentados na Tabela 1 , considerando-se a espécie de triatomíneo, o local de captura e a fonte alimentar. Como dado geral, vê-se que $10(10,6 \%)$ dos 94 triatomíneos foram capturados dentro de casa e $84(89,4 \%)$ no peridomicílio, não havendo diferenças estatisticamente significativas entre $T$. brasiliensis e $T$. pseudomaculata, quanto ao local de captura. 35 insetos (33 T. brasilienses e $2 T$. pseudomaculata) foram capturados na Fazenda Aroeira.

Os marsupiais foram a principal fonte alimentar, seguindo-se as aves, roedores e caprinos. A única diferença significativa foi entre a ornitofilia de $T$. pseudomaculata e a de $T$. brasiliensis, especialmente a nível de peridomicílio. A antropofilia foi baixa $(6,2 \%)$, não havendo diferença entre as duas espécies.

Três insetos da Fazenda Aroeira estavam positivos para $T$. cruzi. Todos eles estavam positivos para marsupial, um deles também para ave e o outro para ave e roedor.

\section{DISCUSSÃO}

A maior ornitofilia de T. pseudomaculata,

Tabela 1 - Fonte alimentar de triatomíneos da Fazenda Aroeira e de quatro localidades proximas.

\begin{tabular}{|c|c|c|c|c|c|c|c|c|c|c|}
\hline \multirow{2}{*}{ Fonte alimentar } & \multicolumn{2}{|c|}{ Intradomicílio } & \multicolumn{2}{|c|}{ Peridomicnlio } & \multicolumn{4}{|c|}{ Subtotais } & \multicolumn{2}{|c|}{ Totais } \\
\hline & TBR & TPS & TBR & TPS & Intr. & $\%$ & Perid. & $\%$ & $N$ & $\%$ \\
\hline Homem & 1 & - & 10 & 2 & 1 & 4,8 & 12 & 6,4 & 13 & 6,2 \\
\hline Marsupial & 4 & 5 & 39 & 35 & 9 & 42,9 & 74 & 39,4 & 83 & 39,7 \\
\hline Ave & 2 & 3 & 17 & 26 & 5 & 23,8 & 43 & 22,9 & 48 & 23,0 \\
\hline Roedor & - & - & 15 & 11 & - & - & $26^{*}$ & 13,9 & 27 & 12,9 \\
\hline Cão & 2 & - & 6 & 2 & 2 & 9,5 & 8 & 4,3 & 10 & 4,8 \\
\hline Gato & 2 & - & 7 & 1 & 2 & 9,5 & 8 & 4,3 & 10 & 4,8 \\
\hline Cabra & 1 & - & 9 & 3 & 1 & 4,8 & 12 & 6,4 & 13 & 6,2 \\
\hline Lagarto & 1 & - & 2 & 1 & 1 & 4,8 & 3 & 1,6 & 4 & 1,9 \\
\hline Não reagente & - & - & 1 & - & - & - & 1 & 0,5 & 1 & 0,5 \\
\hline Soma & 13 & 8 & 106 & 81 & 21 & 100 & $187^{*}$ & 100 & 209 & 100 \\
\hline Total capturado & 4 & 5 & 47 & 37 & 9 & - & $84^{*}$ & - & $93 *$ & \\
\hline
\end{tabular}

* não inclui um $R$. neglectus, possitivo só para roedor.

TBR - T. brasiliensis; TPS - T. pseudomaculata 
Marcondes CB, Dias JCP, Guedes LA, Ferraz Filho AN, Rodrigues VLCC, Mendonça DD. Estudo epidemiológico de fontes de alimentação sangüínea dos triatomíneos da Fazenda Aroeira (Catolé do Rocha, Paraíba) e Circunvizinhanças. Revista da Sociedade Brasileira de Medicina Tropical 24:137-140, jul-set, 1991

no presente estudo, é similar à relatada para o Ceará, conforme análise que fizemos. Desdobrando-se nossos dados, vê-se que esta maior ornitofilia é significativa apenas no peridomicílio. Em estudo de insetos do Piauî̉, nota-se também a alta ornitofilia desta espécie, mas em nenhum destes estudos, ou no de Forattini e cols ${ }^{5}$ para o nordeste, observa-se positividade semelhante para marsupial.

Os marsupiais (provavelmente Didelphis albiventris) são as mais importantes fontes de sangue e de $T$. cruzi para os insetos da área estudada, correspondendo a 39,7\% das fontes alimentares. A baixa positividade para o homem e a variedade de fontes indicam uma pequena adaptação ao domicílio e uma alta mobilidade dos insetos e ressaltam a necessidade de estudos mais aprofundados sobre a transmissão de $T$. cruzi e a biologia de triatomíneos da região, o que também foi ressaltado por Alencar ${ }^{1}$, para o Ceará. Assinalase, ainda, que os caprinos constituíram fonte alimentar de relativa importância na região, acima de gatos e cães. Isto estimula maiores investigações sobre o papel desses animais, já que FuentesCastillo $^{6}$ encontrou $26,1 \%$ de caprinos naturalmente infectados no sul da Paraíba.

A baixa densidade de triatomíneos, associada à baixa positividade para homem, indicam ser muito improvável a infecção deste por contaminação por excreções de triatomíneos associadas à picada. Estas informações, associadas a vários fatores epidemiológicos ${ }^{79}$ apoiam a hipótese de contaminação oral do 26 pacientes detectados no surto da Fazenda Aroeira.

A contaminação oral da tripanosomíase americana acontece habitual mente no ciclo silvestre do T. cruz $i^{2}$, com sua ocorrência no ciclo domiciliar sendo suspeitada em diversos episódios ${ }^{4}$. A importância do surto de Catolé do Rocha é o fato de que o elemento comum aos 26 casos detectados foi sua estada na fazenda num curto período no final de setembro de $1986^{78}$ Os presentes dados, mostrando contato mínimo dos insetos com o homem reforçam hipótese de que a transmissão não tenha ocorrido pelo mecanismo habitual dos triatomíneos, indicando ainda que a infecção natural destes deve-se basicamente a sua associação com marsupiais. Aliás, todos os 11 exemplares de $D$. albiventris da Fazenda Aroeira que examinamos estavam infectados ${ }^{9}$.

\section{SUMMARY}

We have analysed the blood feeding sources of 94 triatomine bugs (51 T. brasiliensis, $42 T$. pseudomaculata and one $R$. neglectus) from Fazenda Aroeira, in Catolé do Rocha Municipality, Paraíba State, and four neighbouring localities. The positivity for marsupial, man and bird blood were, respectively, 39.7,6.2 and 23.0\%. Three insects from Fazenda Aroeira were positive both for T. cruzi and marsupial blood while only one of the three was positive for another mammal. Marsupials are the most important source of $T$. cruzi for the insects of the area, which have litte contact with man.

Key-words: Triatominae. Blood feeding source. Semiarid. Chagas' disease. Northeastern Brazil.

\section{REFERÊNCIAS BIBLIOGRÁFICAS}

1. Alencar JE. História natural da doença de Chagas no Ceará. Fortaleza, Imprensa Univesitária da Universidade Federal do Ceará p.184-185, 1987.

2. Barretto MP. Epidemiologia In: Brener Z, Andrade Z. (eds.) Trypanosoma cruzi e Doença de Chagas. $1^{\text {a }}$ edição, Guanabara Koogan, Rio de Janeiro p.89-151, 1979.

3. Bento DNC, Freitas M, Pinto AS. Epidemiologia da doença de Chagas nos Municípios de Castelo do Piauí e Pedro II, Estado do Piauí, Brasil. Revista da Sociedade Brasileira de Medicina Tropical 22:73-79, 1989.

4. Dias JCP. Mecanismos de transmissão. In: Brener Z, Andrade Z. (eds) Trypanosoma cruzi e Doença de Chagas. 1 a edição, Guanabara Koogan, Rio de Janeiro p.152-174, 1979.

5. Forattini OP, Barata JMS, Santos JLF, Silveira AC. Hábitos alimentares, infecção natural e distribuição de triatomíneos domiciliados na região nordeste do Brasil. Revista de Saúde Pública 15:113-164, 1981 .

6. Fuentes-Castillo AR. Estudio epidemiológico de la infección humana y caprina por Trypanosoma cruzi en el area rural del Município de São Sebastião do Umbuzeiro, Paraíba. Tese de Mestrado, Escola Nacional de Saúde, Rio de Janeiro, 1988.

7. Marcondes CB, Guedes LA, Mendonça D. Surto de doença de Chagas, de provável contaminação oral, em Catolé do Rocha (PB) - observações epidemiológicas. Revista da Sociedade Brasileira de Medicina Tropical 20(supl II):M-11-M-13, 1987. 
Marcondes CB, Dias JCP, Guedes LA, Ferraz Filho AN, Rodrigues VLCC, Mendonça DD. Estudo epidemiológico de fontes de alimentação sangüinea dos triatomíneos da Fazenda Aroeira (Catolé do Rocha, Paraiba) $e$ Circunvizinhanças. Revista da Sociedade Brasileira de Medicina Tropical 24:137-140, jul-set, 1991

8. Shikanai-Yasuda MA. Surto epidêmico de doença de Chagas aguda em Catolé do Rocha, Paraíba. Revista da Sociedade Brasileira de Medicina Tropical 20(supl II):M-14-M-15, 1987.

9. Shikanai-Yasuda MA, Marcondes CB, Guedes LA, Siqueira GS, Barone AA, Dias JCP, Amato Neto V, Tolezano JE, Peres BA, Mendonça DD,
Arruda JrEA, Lopes MH, Shiroma M, Chapadeiro E. Possible oral transmission of acute Chagas' disease in Brazil. Revista do Instituto de Medicina Tropical de São Paulo 33:355-361, 1991.

10. Varejão-Silva MA. Atlas climatológico do Estado da Paraíba. Universidade Federal da Paraíba, Campina Grande, 1984. 